\title{
The Effective Conductivity and the Induced Transmembrane Potential in Dense Cell System Exposed to DC and AC Electric Fields
}

\author{
Mojca Pavlin and Damijan Miklavčič
}

\begin{abstract}
Studying electric potential distribution on the cell membrane and electric conductivity gives us an insight into the effects of the electric field on cells and tissues. Since cells are always surrounded by other cells, we studied how their interactions influence the induced transmembrane potential (TMP) and the effective conductivity in dense cell systems. We numerically and analytically studied the effect of cell organization on the induced TMP and the effective conductivity by organizing cells into simple-cubic, body-centered cubic, and face-centered infinite cubic lattices. We analyzed the general relation between the local quantities (electric field and the induced TMP) and the effective properties such as effective conductivity. We demonstrated that the effective conductivity mainly depends on cell volume fraction, while the induced TMP is affected by cell volume fraction as well as cell ordering. We show that in contrast to some reported results, the phenomenological effective medium theory (EMT) equations cannot be used to determine the local quantities (e.g., the induced TMP) in dense cell systems, whereas the effective properties (e.g., conductivity) can be readily analyzed with EMT equations. We further derive an analytical approximation for the induced TMP in dense cell system exposed to dc and ac electric fields, where dominant factors, which govern the local electric field and the induced TMP, are cell volume fraction and cell ordering. The presented theoretical analysis can be extended also to high frequencies or random distribution of cells.
\end{abstract}

Index Terms-Dense cell system, effective conductivity, frequency domain, numerical methods, transmembrane potential (TMP).

\section{INTRODUCTION}

$\mathbf{T}$ HE INDUCED transmembrane potential (TMP) and electric conductivity of biological cells exposed to electromagnetic fields are of interest in a variety of applications, such as gene electrotransfer [1]-[3], electrochemotherapy [4]-[6], study of forces on cells undergoing fusion [7], [8], models of cardiac tissue response to defibrillating currents [9], and study of potential health effects of electric and magnetic fields [10], [11]. Therefore, investigation of the induced TMP and of the electric conductivity is important in studying the effects of the electric fields on biological cells.

In order to understand how the electric fields interact with cells, we have to evaluate and analyze the induced TMP which

Manuscript received November 14, 2007; revised June 2, 2008. Current version published January 8, 2009. This work was supported by the Slovenian Research Agency under Grants J2-9770 and P2-0249.

The authors are with the Faculty of Electrical Engineering, University of Ljubljana, 1000 Ljubljana, Slovenia (e-mail: mojca.pavlin@ fe.uni-lj.si).

Color versions of one or more of the figures in this paper are available online at http://ieeexplore.org.

Digital Object Identifier 10.1109/TPS.2008.2005292 is generated across the cell membrane [12], [13]. For a single cell exposed to the external (applied) electric field, the analytical calculations of the induced TMP provide very good estimates for cells which are spherical or more general ellipsoidal shapes [12]-[16]. By solving the Laplace equation for a single spherical cell, a general equation can be derived, which describes dependence of the induced TMP on the frequency and electrical properties of the cell and the external medium [12], [17].

The next step for applying these results to real systems is to calculate and analyze dependence of the induced TMP and effective conductivity of cells in dense cell suspensions or tissues. This requires solving Laplace equation for a system of many interacting particles (cells) which, however, cannot be obtained analytically [18]-[20]. In the last few years, it was shown that numerical methods can be successfully applied to obtain the induced TMP in complex cell systems such as suspensions, aggregates, and tissue [21]-[27]. On the other hand, for calculation of the effective conductivity, it was shown by several studies [10], [18], [19], [28] that approximate effective medium theory (EMT) equations can be effectively used to interpret experimental data. In one of the recent studies, Qin et al. [29] proposed that analytical EMT equations could also be used to obtain an analytical approximation for the induced TMP in dense cell systems.

An analytical expression for the induced TMP in dense cell systems would be very useful, since it does not require numerical modeling. Therefore, we tested this hypothesis and more generally analyzed the relation between the local quantities (electric field and the induced TMP) and the effective conductivity of a system of many interacting particles (cells).

We numerically and analytically studied the effect of cell organization on the induced TMP and the effective conductivity by organizing cells into simple-cubic (sc), body-centered cubic (bcc), and face-centered cubic (fcc) lattices. We show that analytical effective medium equations also accurately describe effective conductivity for high density of cells. The effective conductivity of a dense cell suspension does not depend significantly on the organization of the cells for approximately homogeneous ordering of cells. On the contrary, the induced TMP strongly depends on cell ordering. We also show that in contrast to some reported results, the phenomenological EMT equations cannot be used to analytically calculate local electric field and induced TMP in dense systems due to the conceptual difference between effective and local properties. We derive an analytical approximation for the induced TMP for dense cell 


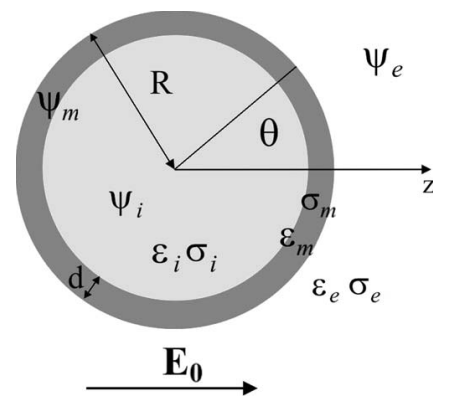

Fig. 1. Schematic representation of a spherical cell, where $\sigma_{\mathrm{e}}, \varepsilon_{\mathrm{e}}, \sigma_{\mathrm{i}}, \varepsilon_{\mathrm{i}}$, and $\sigma_{\mathrm{m}}, \varepsilon_{\mathrm{m}}$ represent specific conductivities and permittivities of the external medium, internal medium, and cell membrane, respectively. $\theta$ is an angle measured with respect to the applied electric field direction $E_{0}, R$ denotes cell radius, and $d$ is the membrane thickness.

system exposed to dc and ac electric fields, where dominant factors which govern local field and induced TMP are cell volume fraction and cell ordering.

\section{THEORY}

\section{A. Induced TMP}

When the electric field is applied to a cell or cell system, a nonuniform TMP is induced on a cell membrane. Potential distribution on the surface of a cell placed in the electric field can be calculated either analytically or numerically.

A simplified model of a biological cell is a sphere consisting of a cell cytoplasm $\sigma_{\mathrm{i}}$ surrounded by a very thin low-conducting membrane $\sigma_{\mathrm{m}}$, which is placed in a conductive medium $\sigma_{\mathrm{e}}$ as shown in Fig. 1, where $d$ denotes membrane thickness, $R$ is the cell radius, and $\theta$ is the angle measured with respect to the electric field direction. Analytical solution for static conditions for the induced TMP is given by Schwan equation [12]-[14]

$$
\Delta \Psi=\mathrm{TMP}=g(\lambda) E_{0} R \cos \theta
$$

where $\Delta \Psi$ represents the potential drop across the cell membrane.

Factor $g(\lambda)$ is a function of cell parameters, and $E_{0}$ is the applied electric field. For physiological conditions where $d \ll R$ and $\sigma_{\mathrm{m}} \ll \sigma_{\mathrm{e}}, \sigma_{\mathrm{i}},(1)$ simplifies in

$$
\Delta \Psi=1.5 R E_{0} \cos \theta .
$$

Equation (2) is a very good approximation for a single spherical cell under physiological conditions, but for very low conductive external media, a more general expression has to be used [12], [14]. An analytical solution can also be derived for spheroidal cells, whereas for arbitrary shaped cells, only numerical methods enable calculations for the induced TMP [30].

Schwan [12], [14] was the first to derive the solution for a spherical cell exposed to ac electric field

$$
\Delta \Psi(j \omega)=\frac{3}{2} R E_{0} \cos \theta \frac{1}{1+j \omega \tau_{\mathrm{m}}}
$$

where time constant $\tau_{\mathrm{m}}$ depends on the electric and geometric properties of a cell

$$
\tau_{\mathrm{m}}=\frac{\varepsilon_{\mathrm{m}}}{\frac{d}{R} \frac{2 \sigma_{\mathrm{e}} \sigma_{\mathrm{i}}}{2 \sigma_{\mathrm{e}}+\sigma_{\mathrm{i}}}+\sigma_{\mathrm{m}}} .
$$

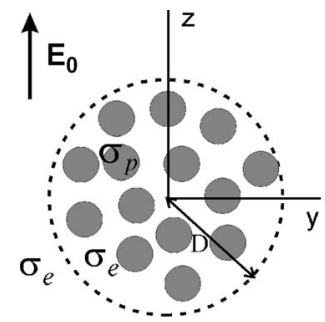

(a)

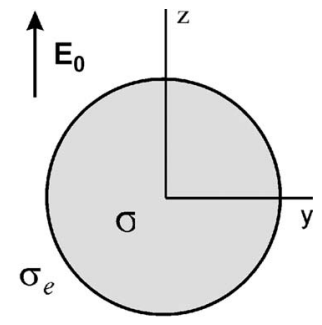

(b)
Fig. 2. Maxwell derivation of the effective conductivity $\sigma$ for a dilute suspension of particles. (a) $N$ spheres having conductivity $\sigma_{\mathrm{p}}$ dispersed in a medium with $\sigma_{\mathrm{e}}$ induce the same potential in the external field $E_{0}$ as (b) one sphere of a radius $D$ having the effective conductivity $\sigma$.

The time constant $\tau_{\mathrm{m}}$ represents a typical time needed for charging of the cell membrane. For a cell exposed to dc electric pulses of duration shorter than $\tau_{\mathrm{m}}$, the cell interior is also exposed to the electric field, resulting in the induced transmembrane voltage across the membrane of the cellular organelles. Thus, for very short high-voltage pulses (nanoseconds), cell organelles can also be permeabilized [31], [32].

\section{B. Effective Conductivity}

The calculation of the effective conductivity of a heterogeneous medium is theoretically a complex problem due to the mutual interactions between the particles. A general theory incorporates all mutual interactions between the particles, with each particle being exposed to a local multipole field which is a sum of all multipole fields of other particles. Since this problem usually cannot be solved, different analytical EMTs which use an average "effective" field were derived [18]-[20], [33]-[36]. For calculation of the effective properties of biological systems, Maxwell EMT equation [34], [35] is most frequently used.

Maxwell derived his EMT equation for the effective conductivity $\sigma$ of a dilute suspension based on a simple example. He calculated potential due to $N$ spheres in an external field, with spheres having conductivity $\sigma_{\mathrm{p}}$ and being dispersed in medium $\sigma_{\mathrm{e}}$ [Fig. 2(a)]. Alternatively, this should be equal to the potential of an equivalent sphere having effective conductivity $\sigma$ [Fig. 2(b)]. With this, he obtained

$$
\frac{\sigma_{\mathrm{e}}-\sigma}{2 \sigma_{\mathrm{e}}+\sigma}=f \frac{\sigma_{\mathrm{e}}-\sigma_{\mathrm{p}}}{2 \sigma_{\mathrm{e}}+\sigma_{\mathrm{p}}}
$$

where $f$ denotes volume fraction of cells dispersed in medium.

Maxwell equation, however, was derived only for dilute suspensions. Bruggeman tried to extend it to concentrated suspensions by a mathematical procedure [18], [20]. He obtained the result known as Bruggeman formula

$$
\frac{\sigma-\sigma_{\mathrm{p}}}{\sigma_{\mathrm{e}}-\sigma_{\mathrm{p}}}\left(\frac{\sigma_{\mathrm{e}}}{\sigma}\right)^{1 / 3}=1-f
$$

which, however, is again an approximation that, only for certain conditions, represents a better approximation from Maxwell equation. Furthermore, for a special case of heterogeneous 
medium with spherical particles arranged in an sc lattice, Rayleigh obtained the following result [33]:

$$
\sigma=\sigma_{\mathrm{e}}\left(1+\frac{3 f}{\frac{\sigma_{\mathrm{p}}+2 \sigma_{\mathrm{e}}}{\sigma_{\mathrm{p}}-\sigma_{\mathrm{e}}}-f-a \frac{\sigma_{\mathrm{p}}-\sigma_{\mathrm{e}}}{\sigma_{\mathrm{p}}+\frac{4}{3} \sigma_{\mathrm{e}}} f^{10 / 3}}\right)
$$

where $a$ is a numerical factor which, according to Rayleigh, is 1.65. Later, Tobias and Meredith, following the same procedure, obtained the same formula with the corrected value of the numerical factor $a$ being 0.523 instead of 1.65 [18], [19].

\section{Equivalent Conductivity of a Single Cell}

For a cell surrounded with a membrane of a conductivity $\sigma_{\mathrm{m}}$ and interior $\sigma_{\mathrm{i}}$, it follows that the field outside the sphere will be the same as the field outside of a homogeneous sphere having an equivalent conductivity $\sigma_{\mathrm{p}}[19]$

$$
\sigma_{\mathrm{p}}=\sigma_{\mathrm{m}} \frac{2(1-\nu) \sigma_{\mathrm{m}}+(1+2 \nu) \sigma_{\mathrm{i}}}{(2+\nu) \sigma_{\mathrm{m}}+(1-\nu) \sigma_{\mathrm{i}}}, \quad \nu=(1-d / R)^{3} .
$$

Under normal physiological conditions, we obtain that cells are practically nonconductive: $\sigma_{\mathrm{p}} \approx 2 \times 10^{-4} \mathrm{~S} / \mathrm{m}$. By introducing the expression for the equivalent conductivity into the Maxwell equation (5) or other EMT equations [18]-[20], the effective conductivity of a cell suspension can be obtained.

\section{Extension to AC Fields-Generalized Conductivity}

The derivations so far are valid only for static conditions, i.e., dc conductivity. To extend these calculations to ac conditions and time-domain problems, the conductivities in (5)-(8) have to be substituted with the complex conductivity defined as [35], [36]

$$
\sigma^{*}=\sigma+j \omega \varepsilon .
$$

However, in many low-frequency measurements of bioelectric phenomena below beta dispersion frequency (approximately $1 \mathrm{MHz}$ ), the second term in the aforementioned equation is much smaller than the first one, so that static analysis is a valid approximation [10], [19].

\section{Methods}

For numerical calculations, we used EMAS (Ansoft Co.) software package, which enables solving partial differential equations for complex geometries using finite element method (FEM). A dc current flow analysis was chosen to calculate the electric potential and current density distribution.

As a model of cell suspension, we used cells ordered in an infinite cubic lattice: sc, bcc, and fcc lattices as shown in Fig. 3.

Biological cells were modeled as nonconductive spheres since, under normal physiological conditions, membrane conductivity is many orders smaller than that of the external medium [10], [37]. Here, the increased membrane conductivity, which occurs during electroporation, was not modeled [38].

By using the symmetry of the cubic lattices and applying appropriate boundary conditions, we were able to model infinite cubic lattices with a model of a unit cell of a given lattice [28].

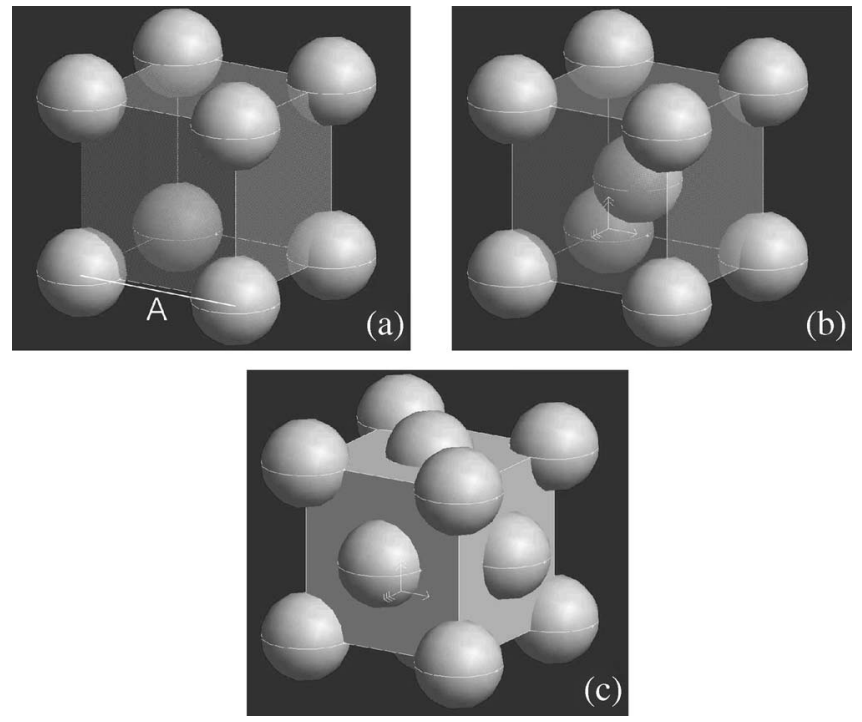

Fig. 3. Unit cells for (a) sc, (b) bcc, and (c) fcc lattices. $A$ is the length of the unit cell side shown in (a). Spheres represent spherical cells ordered in an infinite cubic lattice.

We induced a voltage between surfaces $x y$, thus applying a homogeneous external field. On all other surfaces, the insulation boundary condition was applied (normal components of the currents are zero: $\mathbf{J}_{\mathbf{n}}=0$ ). By changing the ratio between cell radius $R$ and side of the unit cell $A$, we change the volume fraction

$$
f=\frac{4 \pi}{3} N\left(\frac{R}{A}\right)^{3}
$$

where $N$ is the number of spheres contained in each unit cell: $N=1$ (sc), $N=2$ (bcc), and $N=4$ (fcc). From geometries of the unit cells, it follows that the maximum volume fractions for solid spheres are $0.52,0.64$, and 0.74 for sc, bcc, and fcc lattices, respectively.

The induced TMP was determined from the calculated potential distribution, whereas the effective conductivity was obtained from the ratio between the obtained total current and the applied voltage.

\section{REsults}

\section{A. Numerical Results of the Effective Conductivity and of the Induced TMP}

We used FEM to calculate the induced TMP and effective conductivity of cells ordered in infinite cubic lattices (see Fig. 3) which represent a model of a dense cell system such as cell suspensions or tissue. Cells are represented as nonconductive spheres, which is a valid approximation for dc fields and for ac fields below approximately $1 \mathrm{MHz}$ (beta dispersion) [10], [12], [19].

In Fig. 4, we compare the results for the effective conductivity of numerical FEM models to different analytical theories given by (5)-(7). It can be seen that analytical theories for the effective conductivity are exact for smaller values of $f$; however, for higher volume fractions, deviations from numerical values can be observed. In general, the results of our FEM models fit best to Maxwell and Tobias equations. This is in 


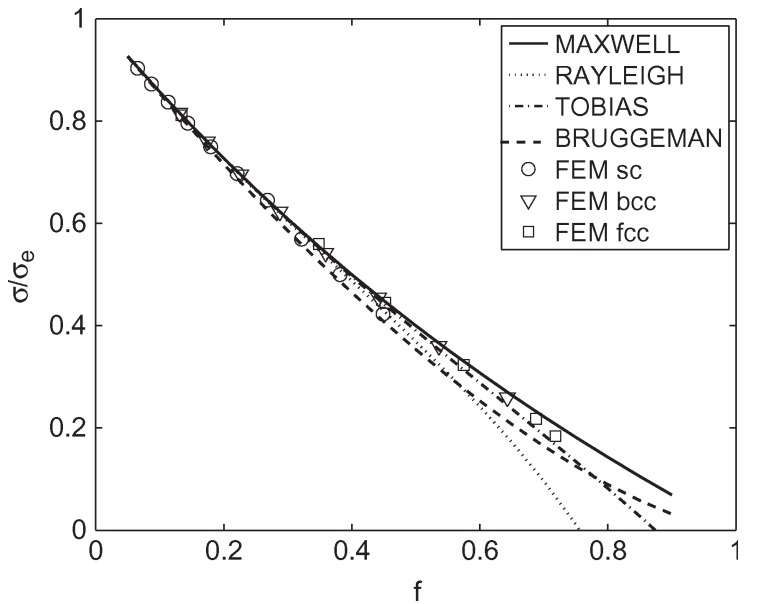

Fig. 4. Normalized effective conductivity of a cell suspension for different volume fraction $f$ of (symbols) numerical FEM results and (lines) analytical EMT solutions of Maxwell, Rayleigh, Tobias, and Bruggeman is shown. Results of FEM calculations were obtained with the models of cells ordered in infinite cubic lattices: sc, bcc, and fcc lattices (see Fig. 3).

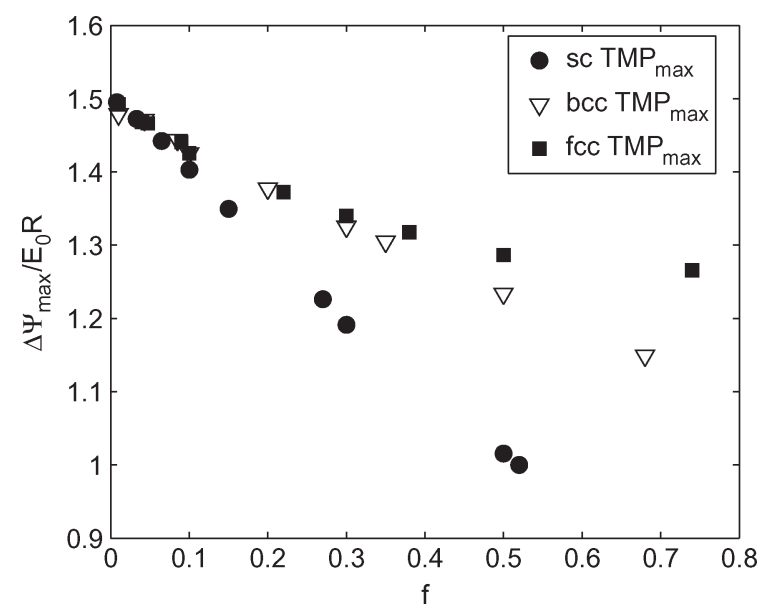

Fig. 5. Comparison of the numerical calculations of the maximal TMP $\Delta \Psi_{\max } / E_{0} R$ of three different cubic lattices (simple cubic-sc, bodycentered cubic-bcc, and face-centered cubic lattice-fcc) for different cell volume fractions $f$.

agreement with the experimental results obtained on different model systems [18], [19], where it was shown that in the case of the ordered spheres of uniform sizes, the experimental results fit best to Maxwell and Tobias equations.

It can also be seen that ordering of cells into different lattices has only minor effect on the effective conductivity for physiological values of parameters where cells are practically nonconductive. Since the volume occupied by the cells does not conduct current, the only parameter that determines the effective conductivity is the cell volume fraction. In other words, the percentage of volume which is conductive mainly determines the effective conductivity of a system of many cells.

In contrast to the effect of ordering on effective conductivity, we can observe (Fig. 5) that the ordering of cells has large effect on the induced TMP in dense cell system. In Fig. 5, numerical results of the normalized maximal TMP $\Delta \Psi_{\max } / E_{0} R$ for sc, bcc, and fcc lattices are compared. For sc and bcc lattices, the induced TMP falls approximately linearly with increasing volume fraction from 1.5 to 1 and 1.15 , respectively, whereas for fcc lattice, it drops more slowly from 1.5 to 1.27 . The largest decrease in induced TMP in dense system is observed for sc lattice (maximum of 30\% decrease), while for fcc, decrease in $\Delta \Psi_{\max } / E_{0} R$ is the smallest (maximum of $15 \%$ ). This can be explained with different ratios of $R / d_{\text {latt }}$ for different cubic lattices, where the parameter $d_{\text {latt }}$ is defined as the distance between the centers of two neighboring cells [see Appendix and Fig. A.1(a)]. Namely in sc lattice, the distances between the cells are smaller for the same volume fraction compared to distances between the cells arranged in fcc lattice consequently leading to larger effect of neighboring cells on the local electric field. The difference between different lattices therefore affects mutual interactions between the cells, and the effect on the local electric field is different.

\section{B. Analytical Calculations of the Induced TMP in Dense Cell System}

Here, we present analytical approximation for the potential induced on the membrane of spherical cells ordered in sc, bcc, and fcc lattices having a given volume fraction of cells $f$ exposed to external ac electric field. The presented equations are valid also for dc fields, taking into account that $\omega=0$.

Following the derivation of Qin et al. [29] (details of the calculations are represented in the Appendix), one obtains the solution for the induced potential on the cell membrane

$$
\Delta \Psi(j \omega)=1.5 \frac{F_{1}(f)}{F_{2}(f, N)} E_{0}(j \omega) R \cos \theta \frac{1}{1+j \omega \tau} .
$$

Factor $F_{1}$ according to Qin et al. [29] reflects the relation between the effective field $E$ and the external field $E_{0}$ and can be derived from Maxwell EMT equation (5)

$$
F_{1}=1+f \frac{\sigma_{\mathrm{e}}^{*}-\sigma_{\mathrm{p}}^{*}}{2 \sigma_{\mathrm{e}}^{*}+\sigma_{\mathrm{p}}^{*}} .
$$

The factor $F_{2}$, as defined in (11), incorporates the change in the potential due to the effect of neighboring cells and depends on cell organization, i.e., the number of nearest neighbors $N$ and the cell volume fraction $f$

$$
F_{2}=1+\left(\frac{3 f}{4 N \pi}\right)^{1 / 3}
$$

where $N=1$ for sc, $N=2$ for bcc, and $N=4$ for fcc lattice.

However, this derivation was based on the assumption that the potential in dense system is affected by the neighboring cells through parameter $F_{2}$ as well as through the change of the electric field, which was derived on the basis of EMT Maxwell equation leading to $F_{1}$. However, this assumption is incorrect since the change in the effective field is already taken into account by the factor $F_{2}$. The factor $F_{1}$, as defined, represents the usual depolarization factor which equals one in an infinite periodic lattice, and therefore, we have to set

$$
F_{1}=1 .
$$

This can be understood also by analyzing the limit case of a very dense system when $f$ is reaching maximal packing ration (0.74 for fcc lattice). Then, the factor $F_{1}$ according to (12) 


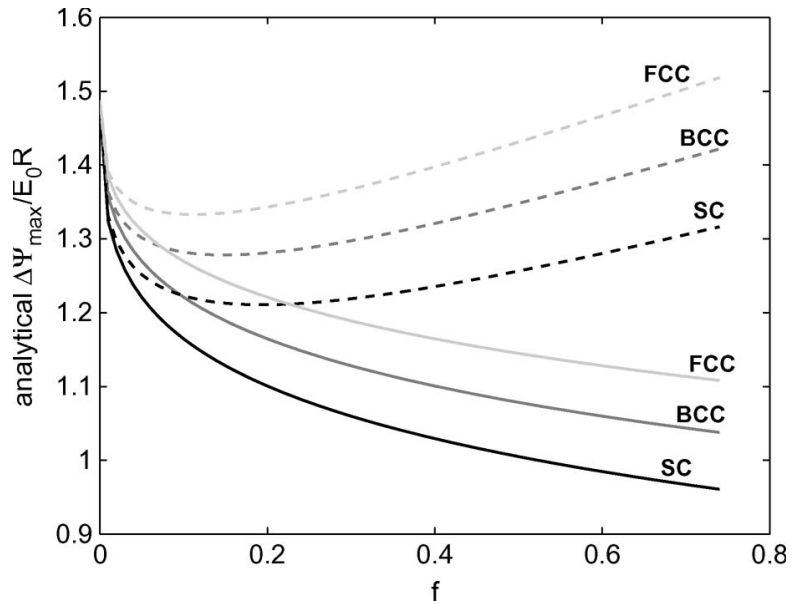

Fig. 6. Comparison of the analytical calculations of the maximal TMP $\Delta \Psi_{\max } / E_{0} R$ for three cubic lattices for dc electric field $(\omega=0)$. We compare our (solid lines) analytical approximation as given in (16) to the (dashed lines) calculations of Qin et al. [29].

would increase to 1.37 , meaning that the induced TMP in a very dense system would be higher then for lower volume fractions, i.e., when $f=0.3$.

From (11)-(14), we have thus finally obtained the analytical approximation for the frequency-dependent induced TMP on cells arranged in cubic lattices

$$
\Delta \Psi(j \omega)=\frac{1.5 E_{0}(j \omega) R \cos \theta}{1+\left(\frac{3 f}{4 N \pi}\right)^{1 / 3}} \frac{1}{1+j \omega \tau} .
$$

The magnitude of the induced TMP exposed to ac field $E_{0}(j \omega)$ is

$$
|\Delta \Psi(j \omega)|=\frac{1}{1+\left(\frac{3 f}{4 N \pi}\right)^{1 / 3}} \frac{1}{\sqrt{1+\left(\omega \tau_{\mathrm{m}}\right)^{2}}} 1.5 E_{0} R \cos \theta .
$$

In Fig. 6, the comparison of the maximal induced TMP calculated using our analytical approximations [see (15)] to the calculations of Qin et al. [see (5) and (6)] is shown for static electric field. It can be seen that, according to Qin et al. [29], the potential should increase for $f>0.1$, which is physically incorrect as well as in disagreement with numerical calculations (see Fig. 5) and experimental results [39]. Our analytical results approximately follow the numerical results for fcc lattice; however, deviations from numerical results for sc and bcc lattices are substantial. The analytical approximation correctly predicts the maximal decrease in $\Delta \Psi_{\max } / E_{0} R$ from 1.5 to 1 for sc lattice as well as basic relation between different organizations as shown in Fig. 5, mainly that $\Delta \Psi_{\max } / E_{0} R \mathrm{sc}<$ bcc $<$ fcc.

In Fig. 7, we calculated the frequency-dependent magnitude of the induced TMP $-\left|\Delta \Psi_{\max }\right| / E_{0} R$ for dense cell system $(f=0.5)$ using analytical approximation (15) and generalized conductivities [see (9)]. To obtain equivalent conductivity from (8), we used average physiological values of conductivities and dielectric constants of membrane, cytoplasm, and external medium given in literature (see Table I). These parameters are constant over a low-frequency range but change above megahertz [17].

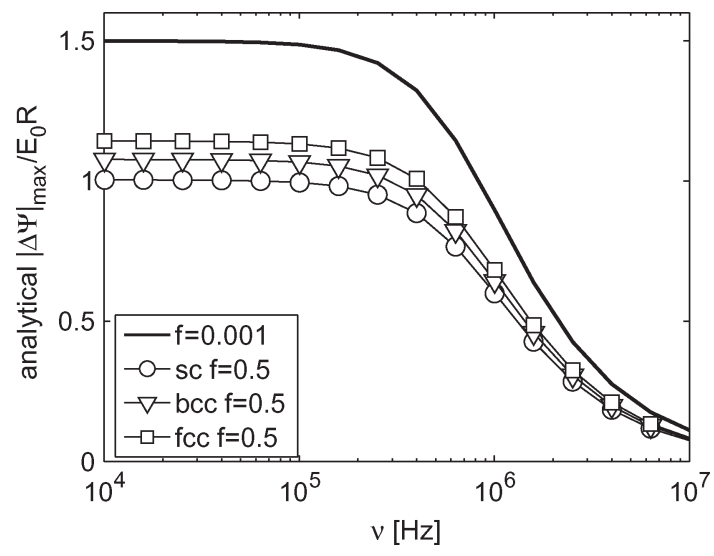

Fig. 7. Frequency dependence of the magnitude of the induced TMP $\left|\Delta \Psi_{\max }\right| / E_{0} R$ obtained analytically from (8), (9), and (15) using the parameters given in Table I. The results are presented for a dense cell system with volume fraction $f=0.5$ for different cell ordering and compared to the results of a single cell (dilute cell suspension) $f=0.001$.

TABLE I

VAlues of Conductivities and PermitTivities From Literature [10], [28] THAT WERE USED IN OUR MODELS

\begin{tabular}{lcc}
\hline & Conductivity & Permittivity \\
\hline external medium & ${ }^{*} \sigma_{\mathrm{e}}=1.2 \mathrm{~S} / \mathrm{m}$ & $\varepsilon_{\mathrm{e}}=7.1 \times 10^{-10} \mathrm{As} / \mathrm{Vm}$ \\
cytoplasm & $\sigma_{\mathrm{i}}=0.5 \mathrm{~S} / \mathrm{m}$ & $\varepsilon_{\mathrm{i}}=7.1 \times 10^{-10} \mathrm{As} / \mathrm{Vm}$ \\
membrane & $\sigma_{\mathrm{m}}=10^{-7} \mathrm{~S} / \mathrm{m}$ & $\varepsilon_{\mathrm{m}}=4.4 \times 10^{-11} \mathrm{As} / \mathrm{Vm}$ \\
\hline membrane thickness & $\mathrm{d}=5 \times 10^{-9} \mathrm{~m}$ \\
cell radius & & $\mathrm{R}=10^{-5} \mathrm{~m}$ \\
\hline
\end{tabular}

*We used $1.2 \mathrm{~S} / \mathrm{m}$ as the value closes to physiological value.

It can clearly be seen that, also for ac electric fields, the induced TMP is decreased for high volume fraction from the values for a single isolated cell (low volume fraction) up to $10 \mathrm{MHz}$, while for higher frequencies, dielectric properties start to dominate and there is no significant effect of cell density on the induced TMP.

\section{DISCUSSION AND CONCLUSION}

In this paper, we present analytical and numerical calculation of the induced TMP and the effective conductivity of cells in dense systems such as cell suspensions and tissue. The presented analysis gives a theoretical evaluation of the interaction of the electric fields in dense cell system by analyzing different volume fractions and cell organizations. These results should offer a better insight of how the electric field interacts with cells in dense systems through the induced TMP and electric current.

By using FEM numerical models, we calculated the effective conductivity and the induced transmembrane voltage of many cell systems where cells were ordered in different cubic lattices. We calculated the effective conductivity for different cell ordering (sc, bcc, and fcc lattices) as well as for different volume fractions of cells. We obtained (see Fig. 4) that the analytical effective medium equations also accurately describe the effective conductivity for high density of cells in agreement with 
experimental studies [10], [19], [38]. We furthermore obtained that the effective conductivity mainly depends only on cell volume fraction (Fig. 4), while cell ordering has no significant effect. In contrast to this, we obtained that the induced TMP is affected by both cell volume fraction and cell ordering, as can be seen in Fig. 5. The numerically calculated decrease in the induced TMP is in agreement with other experimental [39] and numerical [24], [27] studies on dense cell systems.

Since our results clearly show that the effective conductivity does not depend on cell ordering while induced TMP does, this demonstrates that EMT theories cannot be used to calculate local properties such as local electric field or potential since they cannot incorporate ordering of particles in the medium.

We further derived an analytical approximation for the induced TMP in dense cell system exposed to dc and ac electric fields, where dominant factors that govern the local electric field and the induced TMP are the cell volume fraction and cell ordering. Our analytical approximation qualitatively describes the decrease of the induced TMP for cells arranged in different cubic lattices (Figs. 5 and 6 ) and correctly predicts the largest drop of the induced TMP for sc lattice; however, it can only qualitatively describe the dependence on cell volume fraction. The analytical results for cell exposed to ac fields showed that the induced TMP is also decreased for high volume fraction of cell up to $1 \mathrm{MHz}$ (Fig. 7).

More generally, our results are important for all calculations of effective and local properties. We have shown that in contrast to some reported results [29], the phenomenological EMT theories, even though accurate for effective conductivity, cannot be used to determine local fields or potentials in dense cell systems. This is due to the conceptual difference between the theories which calculate local potentials and fields and between the EMT theories which include only average interaction of the neighboring cells. Based on our results, we can conclude that EMT theories can be successfully applied to obtain the effective properties of dense biological systems even for very high volume fraction of cells, while for calculation of the induced TMP, the numerical methods represent the most accurate method.

The presented analysis is relevant for $\mathrm{dc}$ and ac problems for frequencies below $1 \mathrm{MHz}$ where the electric properties of cells are constant and cells behave as nonconductive objects [10], [17]. Nevertheless, numerical FEM models can also be extended to a higher frequency range by taking into account frequency-dependent electrical properties of cells. It should also be stressed that our calculations are valid for cells that are not electrically connected, which is true for cell suspensions [38] and multicellular tumor spheroids [39]. For tissue cells and cells that are electrically connected with gap junctions, other approaches have to be used [22]. Under approximation, which we use, is ordered distribution of cells. In real suspensions, cells order randomly so that cells are as far as possible from each other; therefore, fcc lattice is probably the closest approximation to real cell suspension. On the other hand, in tissue, there is an irregular distribution and/or irregular shape of cells for which different model should be used. In order to extend our analysis to more realistic random or aggregated cell distributions with cells of different sizes, more complex numerical models would have to be used [40].

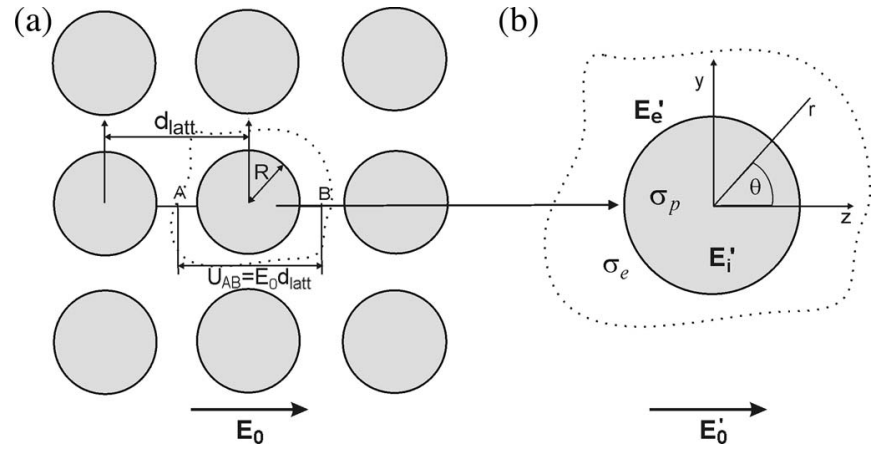

Fig. A.1 (a) Cells ordered in an infinite cubic lattice exposed to the external homogeneous electric field $E_{0}$. (b) Cell inside a dense system can be represented as a single cell exposed to the local field $E_{0}^{\prime}$, which takes into account the effect of neighboring cells. Parameter $d_{\text {latt }}$ is defined as the distance between the centers of two neighboring cells.

\section{APPENDIX}

DERIVATION OF THE ANALYTICAL EXPRESSION FOR THE INDUCED TMP IN DENSE CELL System

First, we follow the derivation of Qin et al. [29] which derives the induced TMP and changes of the local field by taking into account two contributions: the change of the local field due to the changes of the effective field inside a system with many cells with the use of EMTs and the change of the local field due to the effect of surrounding cells.

Biological cells consisting of a cell interior and a cell membrane are replaced with homogeneous spheres having equivalent conductivity, which is valid for dc fields and ac field below $1 \mathrm{MHz}$ [19], [37].

If we assume cubic ordered distribution of biological cells in a suspension, we can evaluate the effect of neighboring cells on the local field by analyzing a unit cell in the cubic lattice. In Fig. A.1, we show cells ordered in an infinite cubic lattice exposed to external applied field $E_{0}$ (a) and a single cell exposed to external local field $E_{0}^{\prime}$. With parameter $d_{\text {latt }}$, we defined the distance between the centers of two neighboring cells. If we assume that the local field $E_{0}^{\prime}$ represents the effect of all neighboring cells, then the single cell exposed to $E_{0}^{\prime}$ represents equivalent to any cell inside an infinite ordered suspension as shown in Fig. A.1(a). From this assumption, it follows that the voltage between $A$ and $B$ along the $z$-axis equals the potential drop along the $z$-axis in Fig. A.1(b)

$$
\begin{aligned}
U_{A B}(j \omega)= & E_{0}(j \omega) \cdot d_{\text {latt }} \\
= & \int_{-d_{\text {latt }} / 2}^{-R} E_{\mathrm{e}}^{\prime}(j \omega) \cdot d z+\int_{-R}^{R} E_{\mathrm{i}}^{\prime}(j \omega) \cdot d z \\
& +\int_{R}^{d_{\text {latt }} / 2} E_{\mathrm{e}}^{\prime}(j \omega) \cdot d z
\end{aligned}
$$

where $E_{\mathrm{e}}^{\prime}$ and $E_{\mathrm{i}}^{\prime}$ denote the local fields outside and inside the single cell as shown in Fig. A.1(b), which is exposed to the local electric field $E_{0}^{\prime}$. From Laplace equation, one can obtain 
the electric field along the $z$-axis inside and outside of a single cell having equivalent conductivity $\sigma_{\mathrm{p}}^{*}$ [Fig. A.1(b)]

$$
\begin{aligned}
& E_{\mathrm{i}}^{\prime}(j \omega)=\frac{3 \sigma_{\mathrm{e}}^{*}}{\sigma_{\mathrm{p}}^{*}+2 \sigma_{\mathrm{e}}^{*}} E_{0}^{\prime}(j \omega) \\
& E_{\mathrm{e}}^{\prime}(j \omega)=\left[2 \frac{\sigma_{\mathrm{p}}^{*}-\sigma_{\mathrm{e}}^{*}}{\sigma_{\mathrm{p}}^{*}+2 \sigma_{\mathrm{e}}^{*}}\left(\frac{R}{r}\right)^{3}+1\right] E_{0}^{\prime}(j \omega) .
\end{aligned}
$$

From (A.1)-(A.3), one can obtain the ratio between the applied electric field $E_{0}$ and the local field $E_{0}^{\prime}$

$$
F_{2}=\frac{E_{0}(j \omega)}{E_{0}^{\prime}(j \omega)}=1+2 \frac{R}{d_{\mathrm{latt}}} \frac{\sigma_{\mathrm{e}}^{*}-\sigma_{\mathrm{p}}^{*}}{\sigma_{\mathrm{p}}^{*}+2 \sigma_{\mathrm{e}}^{*}}
$$

If we take into account that the ratio between $R$ and $d_{\text {latt }}$ depends on the cell volume fraction $f$ and the number of cells in a unit cell $N(N=1$ for sc, $N=2$ for bcc, and $N=4$ for fcc lattice)

$$
\frac{R}{d_{\mathrm{latt}}}=\left(\frac{3 f}{4 N \pi}\right)^{1 / 3}
$$

and using approximation $\sigma_{\mathrm{p}}=0$ (normal physiological conditions), the factor $F_{2}$ simplifies into

$$
F_{2}=1+\left(\frac{3 f}{4 N \pi}\right)^{1 / 3}
$$

According to Qin et al. [29], there is an additional factor $F_{1}$ which changes the local field, which reflects the relation between the effective field $E$ and external field $E_{0}$ and can be derived from Maxwell EMT equation

$$
F_{1}=1+f \frac{\sigma_{\mathrm{e}}^{*}-\sigma_{\mathrm{p}}^{*}}{2 \sigma_{\mathrm{e}}^{*}+\sigma_{\mathrm{p}}^{*}} .
$$

Therefore, according to Qin et al. [29], one can obtain the solution for the induced potential on the cell membrane by taking into account both factors $-F_{1}$ and $F_{2}$

$$
\Delta \Psi(j \omega)=1.5 E_{0}(j \omega) \frac{F_{1}}{F_{2}} R \cos \theta
$$

However, the factor $F_{1}$ represents only the effective field inside a spheroid (aggregate) of spherical particles [24] and is not a valid solution for an infinite lattice. Factor $F_{1}$ is actually the usual depolarization factor which equals one in infinite periodic lattice representing a cell suspension.

From the aforementioned analysis, it therefore follows that the only factor that influences changes in the local field $E_{0}^{\prime}$ and the induced TMP is factor $F_{2}$ [see (A.7)], which incorporates the changes in the local field and the local potential due to the effect of neighboring cells. It depends on cell organization (number of cells in a unit cell $N$ ) and on the cell volume fraction $f$, while $F_{1}=1$.

From this, we obtain that the induced TMP on any cell inside an infinite suspension has to be calculated, assuming that each cell is exposed to the local electric field $E_{0}^{\prime}$. The analytical approximation for the potential induced on cells ordered in infinite cubic lattices gives as the following expression:

$$
\begin{aligned}
\Delta \Psi(j \omega) & =1.5 E_{0}^{\prime}(j \omega) R \cos \theta \\
& =\frac{1.5 E_{0}(j \omega) R \cos \theta}{1+\left(\frac{3 f}{4 N \pi}\right)^{1 / 3}} .
\end{aligned}
$$

Here, we have to stress that this approximation does not take into account higher multipole contributions in the interaction since only dipole factors are taken into account.

\section{REFERENCES}

[1] E. Neumann, M. Schaefer-Ridder, Y. Wang, and P. H. Hofschneider, "Gene transfer into mouse lyoma cells by electroporation in high electric fields," EMBO J., vol. 1, no. 7, pp. 841-845, 1982.

[2] G. J. Prud'homme, Y. Glinka, A. S. Khan, and R. Draghia-Akli, "Electroporation-enhanced nonviral gene transfer for the prevention or treatment of immunological, endocrine and neoplastic diseases," Current Gene Therapy, vol. 6, no. 2, pp. 243-273, Apr. 2006.

[3] M. Cemazar, M. Golzio, G. Sersa, M. P. Rols, and J. Teissié, "Electricallyassisted nucleic acids delivery to tissues in vivo: Where do we stand?" Curr. Pharm. Des., vol. 12, no. 29, pp. 3817-3825, 2006.

[4] M. Okino and H. Mohri, "Effects of a high-voltage electrical impulse and an anticancer drug on in vivo growing tumors," Jpn. J. Cancer Res., vol. 78, no. 12, pp. 1319-1321, Dec. 1987.

[5] L. M. Mir, S. Orlowski, J. Belehradek, Jr., and C. Paoletti, "Electrochemotherapy potentiation of antitumour effect of bleomycin by local electric pulses," Eur. J. Cancer, vol. 27, no. 1, pp. 68-72, 1991.

[6] G. Serša, B. Štabuc, M. Čemažar, B. Jančar, D. Miklavčič, and Z. Rudolf, "Electrochemotherapy with cisplatin: Potentiation of local cisplatin antitumour effectiveness by application of electric pulses in cancer patients," Eur. J. Cancer, vol. 34, no. 8, pp. 1213-1218, Jul. 1998.

[7] K. R. Foster and A. E. Sowers, "Dielectrophoretic forces and potentials induced on pairs of cells in an electric field," Biophys. J., vol. 69, no. 3, pp. 777-784, Sep. 1995.

[8] U. Zimermann, "Electric field-mediated fusion and related electrical phenomena," Biochim. Biophys. Acta, vol. 694, no. 3, pp. 227-277, Nov. 1982.

[9] V. Sharma, F. J. Qu, V. P. Nikolski, P. DeGroot, and I. R. Efimov, "Direct measurements of membrane time constant during defibrillation strength shocks," Heart Rhythm, vol. 4, no. 4, pp. 478-486, Apr. 2007.

[10] K. R. Foster and H. P. Schwan, "Dielectric properties of tissues," in Handbook of Biological Effects of Electromagnetic Fields, C. Polk and E. Postow, Eds. Boca Raton, FL: CRC Press, 1986, pp. 28-96.

[11] T. W. Dawson, M. A. Stuchly, and R. Kavet, "Evaluation of interactions of electric fields due to electrostatic discharge with human tissue," IEEE Trans. Biomed. Eng., vol. 51, no. 12, pp. 2194-2198, Dec. 2004.

[12] E. Neumann, A. E. Sowers, and C. A. Jordan, Electroporation and Electrofusion in Cell Biology. New York: Plenum, 1989.

[13] J. C. Weaver and Y. A. Chizmadzhev, "Theory of electroporation: A review," Bioelectrochem. Bioenerg., vol. 41, no. 2, pp. 135-160, Dec. 1996.

[14] H. P. Schwan, "Electrical properties of tissue and cell suspensions," $A d v$. Biol. Med. Phys., vol. 5, pp. 147-209, 1957.

[15] J. Gimsa and D. Wachner, "Analytical description of the transmembrane voltage induced on arbitrarily oriented ellipsoidal and cylindrical cells," Biophys. J., vol. 81, no. 4, pp. 1888-1896, Oct. 2001.

[16] B. Valič, M. Pavlin, and D. Miklavčič, "The effect of resting transmembrane voltage on cell electropermeabilization: A numerical analysis," Bioelectrochemistry, vol. 63, no. 1/2, pp. 311-315, Jun. 2004.

[17] T. Kotnik and D. Miklavčič, "Second-order model of membrane electric field induced by alternating external electric fields," IEEE Trans. Biomed. Eng., vol. 47, no. 8, pp. 1074-1081, Aug. 2000.

[18] S. S. Dukhin, "Dielectric properties of disperse systems," in Surface and Colloid Science, vol. 3, E. Matijević, Ed. New York: Wiley-Interscience, 1971, pp. 83-165.

[19] S. Takhasima, Electrical Properties of Biopolimers and Membranes. Bristol, U.K.: Adam Hilger, 1989.

[20] T. Hanai, "Electrical properties of emulsions," in Emulsion Science, P. Sherman, Ed. London, U.K.: Academic, 1968, pp. 353-478. 
[21] R. Susil, D. Šemrov, and D. Miklavčič, "Electric field—Induced transmembrane potential depends on cell density and organization," Electro. Magnetobiol., vol. 17, no. 3, pp. 391-399, 1998.

[22] C. E. Fear and M. A. Stuchly, "Biological cells with gap junctions in low-frequency electric fields," IEEE Trans. Biomed. Eng., vol. 45, no. 7, pp. 856-866, Jul. 1998.

[23] C. E. Fear and M. A. Stuchly, "Modeling assemblies of biological cells exposed to electric fields," IEEE Trans. Biomed. Eng., vol. 45, no. 10, pp. 1259-1271, Oct. 1998.

[24] M. Pavlin, N. Pavšelj, and D. Miklavčič, "Dependence of induced transmembrane potential on cell density, arrangement, and cell position inside a cell system," IEEE Trans. Biomed. Eng., vol. 49, no. 6, pp. 605-612, Jun. 2002.

[25] T. R. Gowrishankar and J. C. Weaver, "An approach to electrical modeling of single and multiple cells," Proc. Nat. Acad. Sci. U.S.A., vol. 100, no. 6, pp. 3203-3208, Mar. 2003.

[26] D. A. Stewart, T. R. Gowrishankar, and J. C. Weaver, "Three dimensional transport lattice model for describing action potentials in axons stimulated by external electrodes," Bioelectrochemistry, vol. 69, no. 1, pp. 88-93, Sep. 2006.

[27] A. Ramos, D. O. H. Suzuki, and J. L. B. Marques, "Numerical study of the electrical conductivity and polarization in a suspension of spherical cells," Bioelectrochemistry, vol. 68, no. 2, pp. 213-217, May 2006.

[28] M. Pavlin, T. Slivnik, and D. Miklavčič, "Effective conductivity of cell suspensions," IEEE Trans. Biomed. Eng., vol. 49, no. 1, pp. 77-80, Jan. 2002.

[29] Y. Qin, S. Lai, Y. Jiang, T. Yang, and J. Wang, "Transmembrane voltage induced on a cell membrane in suspensions exposed to an alternating field: A theoretical analysis," Bioelectrochemistry, vol. 67, no. 1, pp. 57-65, Sep. 2005.

[30] G. Pucihar, T. Kotnik, B. Valič, and D. Miklavčič, "Numerical determination of transmembrane voltage induced on irregularly shaped cells," Ann. Biomed. Eng., vol. 34, no. 4, pp. 642-652, Apr. 2006.

[31] A. G. Pakhomov, J. F. Kolb, J. A. White, R. P. Joshi, S. Xiao, and K. H. Schoenbach, "Long-lasting plasma membrane permeabilization in mammalian cells by nanosecond pulsed electric field (nsPEF)," Bioelectromagnetics, vol. 28, no. 8, pp. 655-663, Dec. 2007.

[32] K. H. Schoenbach, S. Xiao, R. P. Joshi, J. T. Camp, T. Heeren, J. F. Kolb, and S. J. Beebe, "The effect of intense subnanosecond electrical pulses on biological cells," IEEE Trans. Plasma Sci., vol. 36, no. 2, pp. 414-422, Apr. 2008.

[33] L. Rayleigh, "On the influence of obstacle arranged in rectangular order upon the properties of a medium," Philos. Mag., vol. 34, pp. 481-502, 1892.

[34] J. C. Maxwell, Treatise on Electricity and Magnetism. London, U.K.: Oxford Univ. Press, 1873.

[35] K. W. Wagner, "Erklärung der dielektrischen Nachwirkungsvorgänge auf Grund Maxwellscher Vorstellungen," Archiv für Elektrotechmik II, vol. 9, pp. 371-387, 1914.
[36] V. H. Pauly and H. P. Schwan, "Über die Impendanc einer Suspension von kugelförmigen teilchen mit einer Schale," Z. Naturforsch., vol. 14b, pp. 125-131, 1959.

[37] M. Pavlin and D. Miklavčič, "Effective conductivity of a suspension of permeabilized cells: A theoretical analysis," Biophys. J., vol. 85, no. 2, pp. 719-729, Aug. 2003.

[38] M. Pavlin, V. Leben, and D. Miklavčič, "Electroporation in dense cell suspension-Theoretical and experimental analysis of ion diffusion and cell permeabilization," Biochim. Biophys. Acta, vol. 1770, no. 1, pp. 12-23, Jan. 2007.

[39] P. J. Canatella, M. M. Black, D. M. Bonnichsen, C. McKenna, and M. R. Prausnitz, "Tissue electroporation: Quantification and analysis of heterogeneous transport in multicellular environments," Biophys. J., vol. 86, no. 5, pp. 3260-3268, May 2004.

[40] B. Drnovšek, V. B. Bregar, and M. Pavlin, "The effect of complex permeability and agglomeration on composite magnetic systems: A threedimensional numerical analysis and comparison with analytical models," J. Appl. Phys., vol. 103, no. 7, p. 07D 924, Apr. 2008.

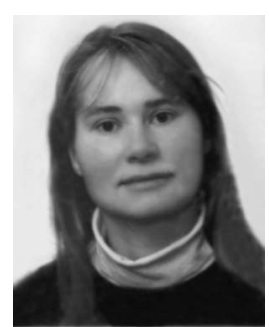

Mojca Pavlin was born in Ljubljana, Slovenia, in 1973. She received the B.S. degree in physics and the Ph.D. degree in electrical engineering from the University of Ljubljana, Ljubljana.

She is currently a Researcher with the Faculty of Electrical Engineering, University of Ljubljana, working on biomedicine and biophysics, particularly the analysis of the interactions of electric fields and biological cells, including numerical modeling and experimental work. Her research interests include electroporation and gene electrotransfer.

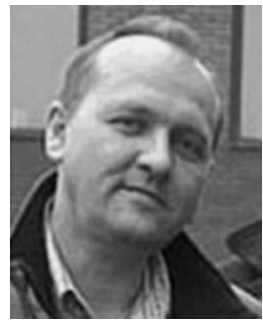

Damijan Miklavčič was born in Ljubljana, Slovenia, in 1963. He received the Ph.D. degree in electrical engineering from the University of Ljubljana, Ljubljana.

$\mathrm{He}$ is currently a Professor with the Faculty of Electrical Engineering and the Head of the Laboratory of Biocybernetics, University of Ljubljana. $\mathrm{He}$ is active in the field of biomedical engineering. His research interest in the last years focuses on electroporation-assisted drug and gene delivery, including cancer treatment by means of electrochemotherapy, tissue oxygenation, and modeling. 